\title{
DISTRIBUIÇÃO GEOGRÁFICA DOS BRACHYURA (CRUSTACEA, DECAPODA) COLETADOS NA PLATAFORMA CONTINENTAL DO NORTE E NORDESTE DO BRASIL
}

\author{
Aline do Vale Barreto ${ }^{1}$ \\ Petrônio Alves Coelho ${ }^{1,3}$ \\ Marilena Ramos-Porto ${ }^{2,3}$
}

\begin{abstract}
GEOGRAPHIC DISTRIBUTION OF BRACHYURA (CRUSTACEA, DECAPODA) COLLECTED ON THE CONTINENTAL SHELF OF NORTH AND NORTHEASTERN BRAZIL. The Brachyura collected on the continental shelf of Northern and Northeastern Brazil understood between the latitudes $4^{\circ} 27^{\prime} \mathrm{N}$ and $3^{\circ} 43^{\prime} \mathrm{S}$ and the longitudes of $50^{\circ} 00^{\prime} \mathrm{W}-38^{\circ} 00^{\prime} \mathrm{W}$, were represented by 133 species, 79 genera and 15 families. The studied material, belongs to the carcinological collection of the Department of Oceanography of University Federal of Pernambuco. The informations obtained in relation to the areas in which these species are found, were gathered from the carcinological files cited above and bibliographic references published until 1991. The majority of the species (125) was found exclusively in the western Atlantic, except three Anfi-Atlantics, two Anfi-Americans, two Circuntropicals and one Indo-Pacific. With regards to the latitude, 11 species were gathered with southern limits in the studies area, 17 species with northern limits in the studied area, six species restricted to the studied area and 99 with northern and southern limits out of the studied area. KEY WORDS. Crustacea, Decapoda, Brachyura, North-northeastern Brazil
\end{abstract}

Os estudos biogeográficos se revestem de grande importância, pois procuram explicar a distribuição dos organismos na superfície dos continentes e oceanos, além de tentar interpretar as causas desta repartição no espaço e no tempo (FURON, 1969; WILEY, 1981).

A maior parte dos conhecimentos sobre a distribuiçào dos seres vivos nos oceanos é relativamente recente, quando comparado com o que se conhece sobre a biota terrestre. Em vista do grande número de comissões oceanográficas realizadas desde os anos de 1965 até 1987 no Norte e Nordeste do Brasil, a região estudada, compreendida entre as latitudes de $4^{\circ} 27^{\prime} \mathrm{N}$ e $3^{\circ} 43^{\prime} \mathrm{S}$ e as longitudes de $50^{\circ} 00^{\prime} \mathrm{W}-38^{\circ} 00^{\prime} \mathrm{W}$, tornou-se uma das mais bem conhecidas no Brasil, permitindo um estudo mais detalhado sobre a ocorrência de organismos marinhos. A presente pesquisa visa, portanto, apresentar uma lista das espécies de crus-

1) Departamento de Oceanografia, Universidade Federal de Pernambuco, Cidade Universitária, 50670-901 Recife, Pernambuco, Brasil.

2) Departamento de Pesca, Universidade Federal Rural de Pernambuco, Cidade Universitária, 50560-901 Recife, Pernambuco, Brasil.

3) Bolsista do CNPq. 
táceos braquiúros encontradas nesta região e estudar sua distribuição geográfica. Do ponto de vista biogeográfico o setor estudado compreende parte das Províncias Guianense e Brasileira (COELHO et al., 1980).

\section{MATERIAL E MÉTODOS}

A coleção carcinológica do Departamento de Oceanografia da Universidade Federal de Pernambuco possui amostras de todas as espécies de braquiúros conhecidas como ocorrendo na plataforma continental do Norte e Nordeste do Brasil. O material estudado foi obtido através de dragagens efetuadas pelo navio oceanográfico "Almirante Saldanha" e pelos barcos "Canopus" e "Pesquisador IV", durante nove comissões oceanográficas, a saber: Canopus, Norte Nordeste I e II, Pesca Norte I, Geomar I, II, III, Pesquisador IV e Pavasas. As informações sobre a área total de ocorrência de cada espécie foram extraídas da literatura pertinente, complementadas pelos dados do acervo da coleção acima mencionada.

Foram estudadas as distribuições longitudinal e latitudinal de cada espécie. Com relação à distribuição longitudinal foram consideradas as áreas do Pacífico Oriental, Atlântico Ocidental, Atlântico Oriental e Indo-Pacífico. A distribuição latitudinal foi estudada apenas no Atlântico Ocidental, sendo referida por abreviaturas, todas as unidades geográficas representando os estados pertencentes ao leste dos Estados Unidos e ao Brasil e os países nos demais casos. Todos os estados, entretanto, que formam a parte Norte do Golfo do México, assim como todos os países das Antilhas e do leste da América Central, foram reunidos em unidades geográficas. A península de Yucatan, por sua vez, foi referida isoladamente, assim como as Bermudas, o Arquipélago de Fernando de Noronha e o Atol das Rocas.

A distribuição longitudinal é mostrada em figuras e a latitudinal em tabelas. É utilizada a nomenclatura adotada por MELLO (1985) para a distribuição longitudinal das espécies.

\section{RESULTADOS E DISCUSSÃO}

A fauna braquiurológica da região está representada por 133 espécies, distribuídas em 70 gêneros e 15 familias, cuja distribuição apresentada na tabela I é resultado de coletas aqui realizadas e sobretudo de dados extraídos de VERRIL (1908), RATHBUN (1918, 1925, 1930, 1937), MONOD (1956), GARTH (1958), GUINOT-DUMORTIER (1959, 1960), HOLTHUIS (1959), RICHMOND (1962), BULLIS \& THOMPSON (1965), GUNTER \& HALL (1965), WILLIAMS (1965, 1984), FAUSTO-FILHO (1966, 1967, 1968, 1970, 1975, 1978, 1979), PEQUEGNAT (1970), PEQUEGNAT et al. (1971), FRANKS et al. (1972), POWERS (1977), COELHO \& BARRETO (1990), COELHO \& RAMOS PORTO (1980, 1986, 1987/89), COELHO et al. (1980), GOMES-CORRÊA \& SILVA-BRUM (1980), GOUVÊA (1980, 1986), RODRIGUEZ (1980), SOTO (1980), RODRIGUEZ (1982), MELO (1985, 1987), 


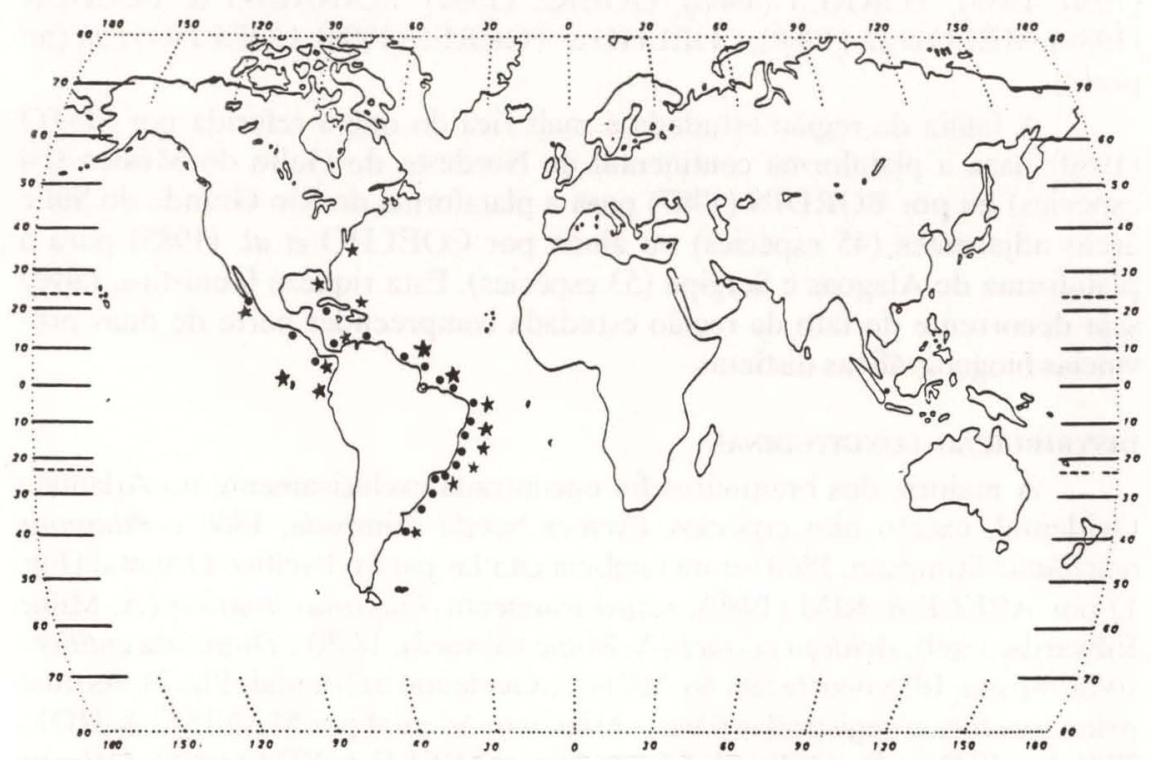

Fig. 1. Distribuição de Cycloes bairdii (*) e Pilumnus reticulatus (•).

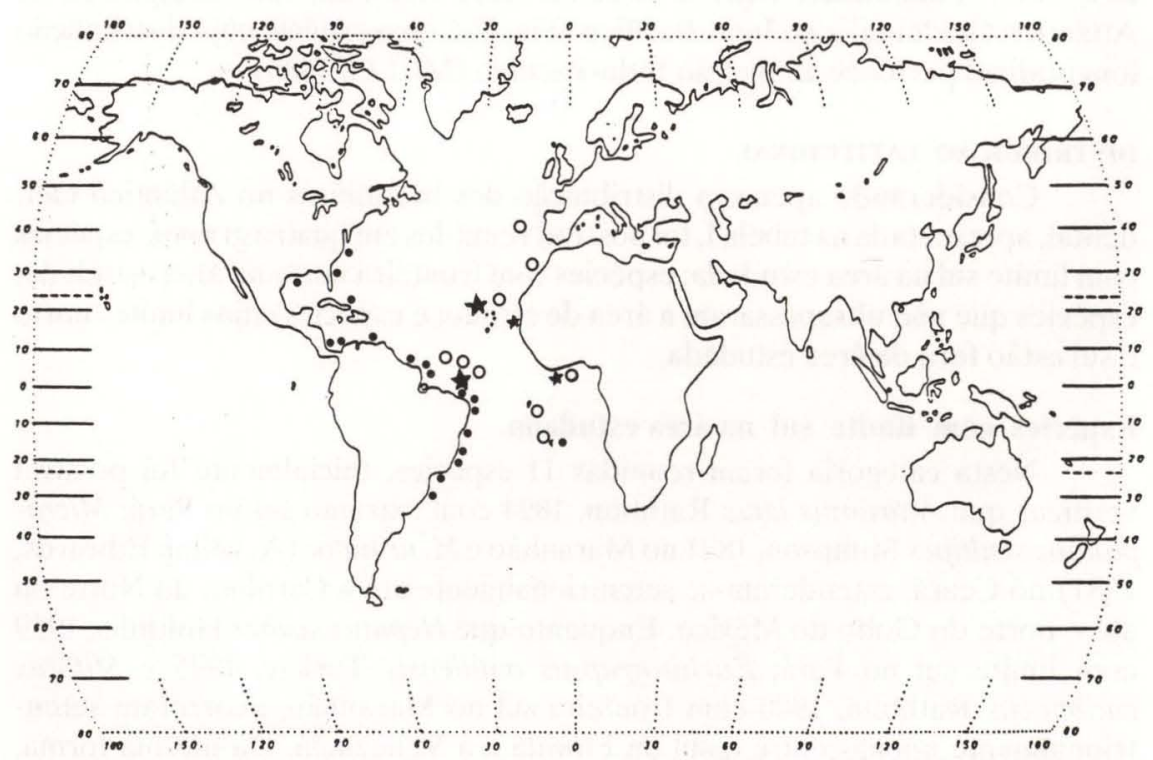

Fig. 2. Distribuição de Dromidia antillensis (•), Euryozius bouvieri (0) e Acidops cessaci (*). 
MARTÍNEZ-IGLESIAS \& GOMES-HERNÁNDEZ (1986), ABELE \& KIM (1986, 1989), TORRES (1988), GOEKE (1989), MANNING \& FELDER (1989), MELO et al. (1989), COELHO \& TORRES (1990), COELHO et al. (no prelo).

A fauna da região estudada é mais rica do que a referida por SOTO (1980) para a plataforma continental do Nordeste do Golfo do México (64 espécies) ou por BORDIN (1987) para a plataforma do Rio Grande do Sul e áreas adjacentes (45 espécies) ou ainda por COELHO et al. (1983) para a plataforma de Alagoas e Sergipe (53 espécies). Esta riqueza faunística, talvez seja decorrente do fato da região estudada compreender parte de duas províncias biogeográficas distintas.

\section{DISTRIBUIÇÃO LONGITUDINAL}

A maioria dos braquiúros foi encontrada exclusivamente no Atlântico Ocidental, exceto oito espécies. Cycloes bairdii Stimpson, 1860 e Pilumnus reticulatus Stimpson, 1860 foram também citadas para o Pacífico Oriental (Fig. 1) por ABELE \& KIM (1989), respectivamente. Euryozius bouvieri (A. Milne Edwards, 1869), Acidops cessaci (A. Milne Edwards, 1878) e Dromidia antillensis Stimpson, 1858 ocorreram no Atlântico Ocidental e Oriental (Fig. 2). As duas primeiras foram registradas para o Atlântico Oriental por MANING \& HOLTHUIS (1981) e D. antillensis foi citada por ABELE \& KIM (1986). Calappa gallus (Herbst, 1803) que apresentou registro no Atlântico e no Indo-Pacífico e Cronius ruber (Lamarck, 1818) que ocorreu no Atlântico Oriental e Ocidental e no Pacífico Oriental (Fig. 3), foram consideradas como Circuntropicais (MELO, 1985). Finalmente, Thyrolambrus astroides Rathbun, 1894 coligida só no Atlântico Ocidental e no Indo-Pacífico (Fig. 4) é uma espécie cuja distribuição longitudinal pertence ao padrão Indo-Pacífico (MELO, 1985).

\section{DISTRIBUIÇÃO LATITUDINAL}

Considerando apenas a distribuição dos braquiúros no Atlântico Ocidental, apresentada na tabela I, foi possível reuní-los em quatro grupos: espécies com limite sul na área estudada; espécies com fronteira norte na área estudada; espécies que não ultrapassaram a área de estudo; e espécies cujos limites norte e sul estão fora da área estudada.

\section{Espécies com limite sul na área estudada.}

Nesta categoria foram reunidas 11 espécies. Inicialmente foi possível verificar que Anasimus latus Rathbun, 1894 com extremo sul no Pará; Micropanope scultipes Stimpson, 1871 no Maranhão e M. urinator (A. Milne Edwards, 1881) no Ceará, estenderam-se setentrionalmente até a Carolina do Norte ou até o norte do Golfo do México. Enquanto que Hepatus scaber Holthuis, 1959 com limite sul no Pará; Euchirograpsus antillensis Turkay, 1975 e Mithrax caribbaeus Rathbun, 1900 com fronteira sul no Maranhão, ocorreram setentrionalmente apenas entre o sul da Flórida e a Venezuela. Da mesma forma, Cyclopax pinnotheroides Guinot, 1969, Portunus rufiremus Holthuis, 1959, duas 


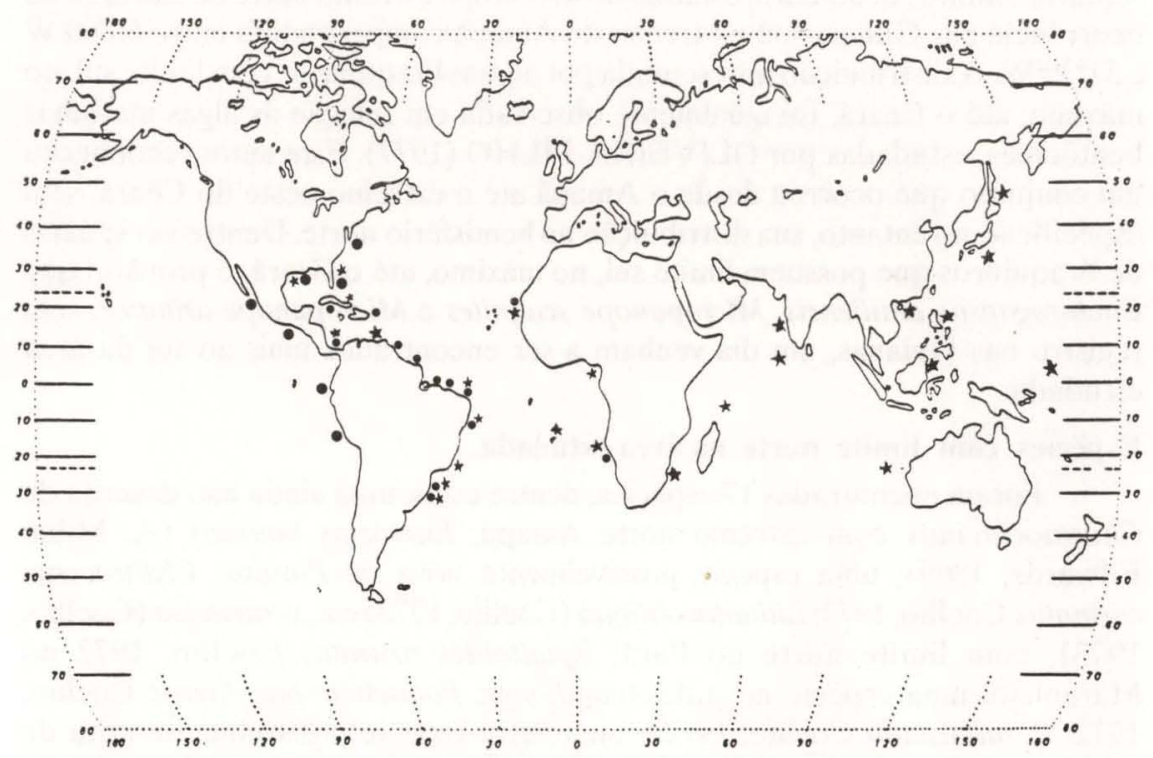

Fig. 3. Distribuiç̧ão de Calappa gallus $\left(^{*}\right)$ e Cronius ruber $(\bullet)$.

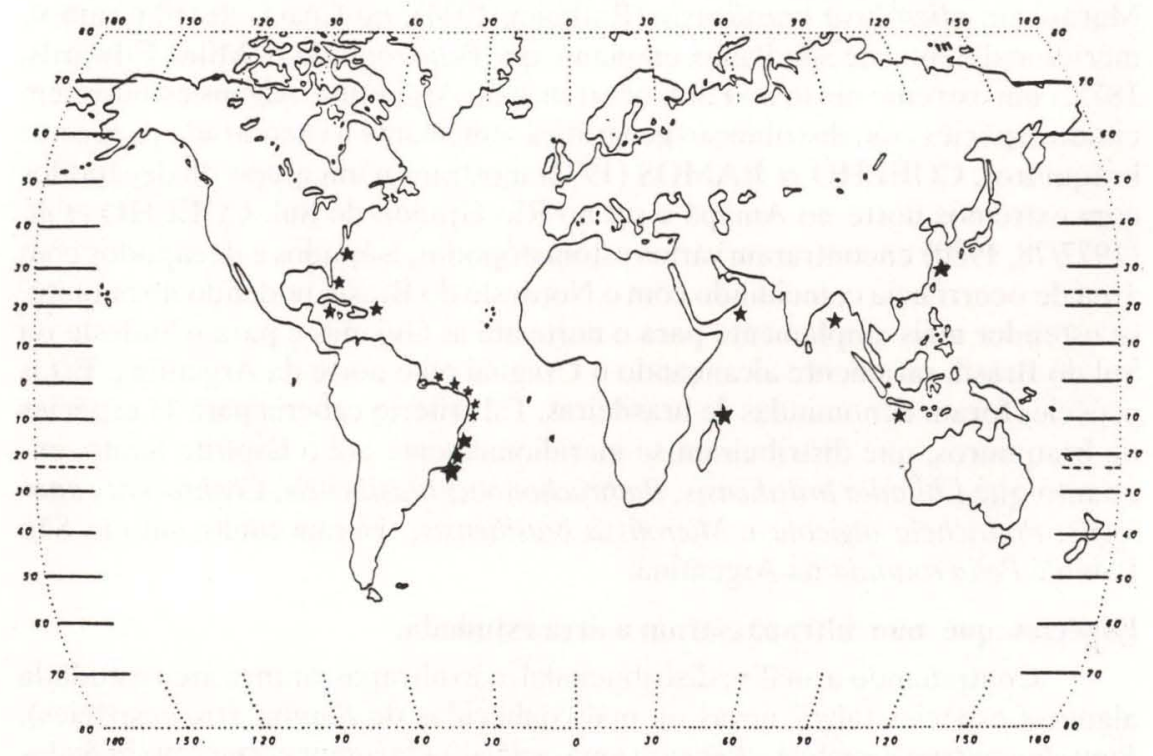

Fig. 4. Distribuição de Thyrolambrus astroides (*). 
espécies provavelmente novas de Chasmocarcinus e uma de Palicus apresentaram limite sul no Pará, estando no entanto, o extremo norte de sua área de ocorrência nas Guianas ou no trecho do Amapá compreendido entre $50^{\circ} 00^{\prime} \mathrm{W}$ e 51³0'W. A distribuição apresentada por alguns braquiúros, com limite sul, no máximo, até o Ceará, foi igualmente observada em relação às algas marinhas bentônicas, estudadas por OLIVEIRA-FILHO (1977). Este autor reconheceu um conjunto que ocorreu desde o Amapá até o extremo oeste do Ceará, sem especificar, no entanto, sua distribuição no hemisfério norte. Dentre as espécies de braquiúros que possuem limite sul, no máximo, até o Ceará, é provável que Euchirogapsus antillensis, Micropanope sculptites e Micropanope urinator, sem registro nas Guianas, um dia venham a ser encontrados mais ao sul da área estudada.

\section{Espécies com limite norte na área estudada.}

Foram encontradas 17 espécies, dentre estas, uma ainda não descrita de Chasmocarcinus com extremo norte Amapá; Euryozius bouvieri (A. Milne Edwards, 1869); uma espécie possivelmente nova de Pinnixa; Clythrocerus carinatus Coelho, 1973; Lithadia obliqua (Coelho, 1973) e L. vertiginosa (Coelho, 1973), com limite norte no Pará; Epialtoides rostratus Coelho, 1972 no Maranhão; uma espécie nova de Acanthonyx; Podochela brasiliensis Coelho, 1972; P. minuscula Coelho, 1972 e uma outra espécie possivelmente nova de Chasmocarcinus com fronteira norte no Ceará, estenderam-se meridionalmente, no máximo até o Espírito Santo. Por outro lado, Lithadia brasiliensis (Martens, 1872) e Batrachonatus brasiliensis Rathbun, 1894, com limite norte no Pará; Clythrocerus analogus Coelho, 1973 e Podochela algicola Stebling, 1914 no Maranhão; Microlissa brasiliensis (Rathbun, 1924), no Ceará, distribuiram-se meridionalmente até São Paulo, enquanto que Pelia rotunda A. Milne Edwards, 1875, com extremo norte no Pará, ocorreu até a Argentina. Alguns estudos têm citado espécies com distribuição geográfica semelhante à encontrada para estes braquiúros. COELHO \& RAMOS (1972) mostraram um grupo de decápodos com extremos norte no Amapá e sul no Rio Grande do Sul. COELHO et al. $(1977 / 78,1980)$ encontraram vários estomatópodos, isópodos e decápodos com área de ocorrência coincidindo com o Nordeste do Brasil, podendo no entanto, se estender mais amplamente para o norte até as Guianas e para o Sudeste ou Sul do Brasil, raramente alcançando o Uruguai ou o norte da Argentina. Estas espécies foram denomindas de brasileiras. Tal critério caberia para 11 espécies de braquiúros, que distribuiram-se meridionalmente até o Espírito Santo, enquanto que Lithadia brasiliensis, Bathrachonotus brasiliensis, Clythrocerus analogus, Podochela algicola e Microlissa brasiliensis, tiveram limite sul em São Paulo e Pelia rotunda na Argentina.

\section{Espécies que não ultrapassaram a área estudada.}

Continuando a análise distribucional, não ultrapassaram a área estudada algumas espécies talvez novas ou mal conhecidas de Pinnixa (três espécies), Pinnotheres (uma espécie), Palicus (uma espécie) e Acidops cessaci, assinaladas para uma única localidade, dificultando sua comparação com outros grupos. 
Tabela I. Distribuição latitudinal dos Brachyura (Crustacea, Decapoda) coletados na plataforma continental Norte e Nordeste do Brasil $\left(50^{\circ} \mathrm{W}-18^{\circ} \mathrm{W}\right)$. (MS) Massachusetts, (NJ) Nova Jersey, (Del) Delaware, (VA) Virgínia, (CM) Carolina do Norte, (CS) Carolina do Sul, (Fla) Sudeste e Sudoeste da Flórida (EUA), (BM) Bermudas, (GMx) Norte do Golfo do México, (Yu) Yucatan-Mx, (AC) Costa Atlântica da América Central, (COL) Colômbia, (VEN) Venezuela, (T) Trinidad, (GUI) Guianas, (AP) Amapá, (PA) Pará, (MA) Maranhão, (PI) Piauí, (CE) Ceará, (RN) Rio Grande do Norte, (PB) Paraiba, (PE) Pernambuco, (AL) Alagoas, (SE) Sergipe, (BA) Bahia, (ES) Espírito Santo, (RJ) Rio de Janeiro, (SP) São Paulo, (PR) Paraná, (SC) Santa Catarina, (RS) Rio Grande do Sul, (FN) Arquipélago de Fernando de Noronha, (R) Atol das Rocas (Brasil), (URU) Uruguai, (ARG) Argentina.

Táxons

Atlântico Ocidental

DROMIIDAE

Dromidia antillensis Stimpson, 1858 CN-Fla-BM-GMx- A- AC- COL-VEN-GUI- AP-PA-

MA-PI-CE-RN-PB-PE-AL-BA-ES-RJ-SP-SC-RS

Hypoconcha arcuata Stimpson, 1858

H. parasitica (Linnaeus, 1763)

CN-FLA-GMx- A-GUI- AP-PA-PB-SE-BA-ES-RJ-SP

CN-FLA-GMx-A-VEN-GUI-AP-PA-MA-CE-RN-PBPE-AL-BA-ES-SP-SC

\section{RANINIDAE}

Raninoides loevis (Latreille, 1825)

CN-FLA-GMx-A-GUI-AP-PA-MA-PI-CE-RN-PB-PEAL-BA-SP

Symethis variolosa (Fabricius, 1793)

CN-Fla-A-AP-PA-MA-CE-RN-PB-PE-AL-SE-BA-RJ$\mathrm{RN}$

\section{DORIPPIDAE}

Clythrocerus analogus Coelho, 1973

C. carinatus Coelho, 1973

MA-PE-ES-SP

C. stimpsoni Rathbun, 1937

PA-MA-PI-PE

Ethusa americana A. Milne

Fla-AP-RJ-RS

CN-Fla-A-MA-PI-CE-PE-AL-SE-BA-RJ-SP

Edwards, 1880

CALAPPIDAE

Calappa gallus (Herbst, 1803)

Fla-GMx-A-AC-VEN-MA-CE-RN-PE-AL-BA-RJ-SPRS-R

C. ocellata Holthuis, 1958

CN-Fla-BM-A-AC-COL-VEN-AP-PA-MA-CE-RN-PBPE-BA-RJ-R

Cycloes bairdii Stimpson, 1860

CN-Fla-BM-A-COL-GUI-AP-PA-MA-PI-CE-RN-PB-P E-AL-BA-ES-RJ-R-ARG

\section{LEUCOSIIDAE}

Callidactylus asper Stimpson, 1871

CN-Fla-GMx-A-AP-PA-MA-CE-RN-PB-AL

Ebalia stimpsoni A. Milne Edwards, 1880

CN-Fla-A-COL-AP-PA-MA-PI-CE-RN-PB-PE-A-BA-

RJ-SP

Iliacantha intermedia Miers, 1886

CN-Fla-GMx-A-VEN-MA-PE-BA

I. liodactylus Rathbun, 1898

I. sparsa Stimpson, 1871

Fla-A-T-GUI-AP-TA-AL-SE-BA

Fla-A-PA-MA-CE-RN-PE-AL-SE-BA-RJ

I. subglobosa Stimpson, 1871

CN-FLA-GMx-A-AP-PA-MA-RN-PB-AL

Lithadia brasiliensis (Martens, 1872) PA-PE-SE-RI-SP

L. conica (Coelho, 1973)

L. obliqua (Coelho, 1973)

AP-PA-MA-PI-CE-RN-PE-ES

PA-CE-RN-PE

L. vertiginosa (Coelho, 1973)

PA-MA-CE-PB-PE-AL-BA

Cont. 
Tabela I. (Cont.)

Táxons

Persephona crinita Rathbun, 1931

P. finneganae Rathbun, 1937

P. lichtensteinii Leach, 1817

P. punctata (Linnaeus, 1758)
Atlântico Ocidental

Fla-GMx-A-T-GUI-AP-PA-RJ-PR-SC

A-GUI-AP-PE-AL-SE-RJ-SP

VEN-GUI-AP-PA-MA-PE-AL-SE-ES-RJ-SP-SC

A-AC-GUI-AP-PA-CE-PE-AL-SE-RJ-SP-PR-RS

Speloeophorus elevatus Rathbun, 1898 Fla-A-MA-CE-RN-PB-PE-AL-BA

\section{MAJIDAE}

Acanthonyx sp.

Aepinus septemspinosus (A. Milne Edwards, 1879)

Anasimus fugax (A. Milne Edwards, 1880)

A. latus Rathbun, 1894

Arachnopsis filipes Stimpson, 1871

Batrachonotus brasiliensis Rathbun, 1894

Chorinus heros (Herbst, 1790)

Collodes armatus Rathbun, 1898

C. inermis A. Milne Edwards, 1878

C. trispinosus Stimpson, 1871

Epialtoides rostratus Coelho, 1972

Euprognatha acuta A. Milne Edwards, MS-Fla-GMx-A-GUI-AP-PA-PE-RJ-SP-SC-RS-URU 1880

E. gracilipes A. Milne Edwards, 1878

Hemus cristulipes A. Milne Edwards, 1875

Herbstia depressa Stimpson, 1860

Inachoides forceps A. Milne Edwards, 1879

Leptopisa setirostris (Stimpson, 1871)

Libinia ferreirae Brito Capello, 1871

Macroeloma concavum (Miers, 1886)

M. eutheca (Stimpson, 1871)

M. laevigatum (Stimpson, 1860)

M. septemspinosum (Stimpson, 1871)

M. trispinosum (Latreille, 1825)

Microlissa brasiliensis (Rathbun, 1924) CE-PE-BA-ES-RJ-SP

Microphrys interruptus Rathbun, 1920 A-COL-MA-PI-CE-RN-PB-PE-AL-ES-RJ-SP-FN-R

Mithraculus forceps A. Milne

Edwards, 1875

Mithrax caribbaeus Rathbun, 1900

M. hemphilli Rathbun, 1892
Fla-YU-A-GUI-AP-PA-MA-CE-RN-PB-PE-AL-ES-RJSP

\section{CE-RN-PE-BA}

CN-Fla-GMx-A-COL-VEN-PA-CE-RN-PB-PE-AL-BA -RJ-SP-FN-R

BM-A-AP-RJ

CN-Fla-GMx-COL-VEN-T-GUI-AP-PA

CN-Fla-GMx-A-AP-PA-CE-RN

PA-MA-PI-CE-RN-PB-PE-AL-BA-RJ-SP

Fla-BM-YU-A-COL-VEN-MA-CE-RN-PB-PE-AL-SEBA-ES-R

A-PA-MA-ES-SP

A-COL-GUI-AP-PA-MA-PI-CE-RN-PB-PE-AL-BA

CN-Fla-GMx-A-AP-PA-SP

MA-PI-RN-PB-PE-AL-ES

CN-GMx-A-AC-COL-VEN-MA-CE-RN-PE-FN

COL-VEN-MA-AL

CN-Fla-GMx-A-COL-VEN-GUI-AP-PA-MA-CE-RN

-PE-BA-RJ-SP-SC

Fla-A-VEN-MA-PI-CE-RN-PB-PE-AL-BA-ES

COL-VEN-GUI-AP-PA-PI-RN-PB-PE-AL-SE-RJ-SPPR-SC-URU-ARG

A-AC-PA-MA-PI-CE-RN-PB-PE-AL-BA-FN

CN-Fla-GMx-A-MA-PI-CE-RN-PB-PE-AL-BA-ES

Fla-A-COL-PI-CE-RN-PB-PE-AL

CS-Fla-GMx-A-GUI-CE-RN-PB-PE-AL-BA

CN-Fla-BM-GMx-YU-A-COL-VEN-PA-MA-PI-CE-

RN-PB-PE-AL-BA-ES-SP

CN-Fla-BM-GMx-A-COL-VEN-T-GUI-MA-PI-CE-

RN-PB-PE-AL-BA-RJ-SP-SC-FN-R

A-COL-VEN-GUI-PA-MA

Fla-A-COL-VEN-MA-CE-PB-PE-AL-BA-RJ-R

Cont. 
Tabela I. (Cont.)

\begin{tabular}{|c|c|}
\hline Táxons & Atlântico Ocidental \\
\hline Mithrax hispidus (Herbst, 1790) & $\begin{array}{l}\text { Del-Fla-BM-GMx-A-VEN-GUI-PA-MA-CE-RN-PB- } \\
\text { PE-AL-BA-ES-RJ-SP }\end{array}$ \\
\hline $\begin{array}{l}\text { Mocosoa crebripunctata Stimpson, } \\
1871\end{array}$ & Fla- GMx-MA-CE-RN-PB-PE-ES-RJ \\
\hline Nemausa acuticornis Stimpson, 1871 & $\begin{array}{l}\text { CN-Fla-GMx-Yu-A-AP-PA-MA-PI-CE-RN-PB-PE-AL- } \\
\text { SE-BA-ES-RJ-SP-FN }\end{array}$ \\
\hline N. cornutus (Saussure, 1857) & Fla-BM-GMx-A-VEN-AP-PA-MA-RN-BA-ES-R \\
\hline Notolopas brasiliensis Miers, 1886 & GUI-AP-PA-MA-CE-RN-PB-PE-AL-SE-BA-RJ-SP-PR \\
\hline \multicolumn{2}{|c|}{$\begin{array}{l}\text { Paradasygyus tuberculatus (Lemos de GUI-AP-PA-CE-RN } \\
\text { Castro, 1949) }\end{array}$} \\
\hline Pelia rotunda A. Milne Edwards, 1875 & $\begin{array}{l}\text { PA-MA-CE-RN-PB-PE-AL-BA-RJ-PR-SC-RS-URU- } \\
\text { ARG }\end{array}$ \\
\hline Picroceroides tubularis Miers, 1886 & Fla-A-AP-PA-MA-CE-RN-PB-PE-AL-BA-ES-FN \\
\hline Pitho Iherminieri (Schramm, 1867) & $\begin{array}{l}\text { CN-Fla-GMx-A-COL-VEN-PA-MA-PI-CE-RN-PB-PE- } \\
\text { AL-BA-ES-RJ-SP-FN-R }\end{array}$ \\
\hline $\begin{array}{l}\text { Podochela (Coryrhynchus) algicola } \\
\text { Stebling, } 1914\end{array}$ & MA-CE-RN-PB-PE-AL-BA-ES-RJ-SP \\
\hline $\begin{array}{l}\text { P. (Podochela) brasiliensis Coelho, } \\
1972\end{array}$ & CE-PB-PE-SE-BA \\
\hline $\begin{array}{l}\text { P. (Ericerodes) gracilipes Stimpson, } \\
1871\end{array}$ & $\begin{array}{l}\text { CN-Fla-GMx-Yu-A-COL-AP-PA-MA-PI-CE-RN-BA- } \\
\text { ES-RJ-SP-PR-SC-RS }\end{array}$ \\
\hline P. (E.) minuscula Coelho, 1972 & CE-RN-PE-BA \\
\hline $\begin{array}{l}\text { Stenorhynchus seticornis (Herbst, } \\
1788 \text { ) }\end{array}$ & $\begin{array}{l}\text { CN-Fla-GMx-A-AC-COL-VEN-GUI-AP-PA-MA-PI-CE } \\
\text {-RN-PB-AL-SE-BA-ES-RJ-SP-SC-RS }\end{array}$ \\
\hline $\begin{array}{c}\text { PARTHENOPIDAE } \\
\text { Cryptopodia concava Stimpson, } 1871\end{array}$ & $\begin{array}{l}\text { CN-Fla-GMx-A-AP-MA-PI-CE-RN-PB-PE-AL-BA-ES- } \\
\text { RJ }\end{array}$ \\
\hline Hepatus gronovii Holthuis, 1959 & COL-VEN-GUI-AP-PA-MA-RJ-SC \\
\hline H. scaber Holthuis, 1959 & VEN-GUI-AP-PA \\
\hline Heterocrypta lapidea Rathbun, 1901 & A-PA-MA-CE-RN-PE-AL-SE-BA-RJ-SP-SC-RS \\
\hline Leiolambrus nitidus Rathbun, 1901 & GMx-A-VEN-GUI-AP-PA-SE-ARG \\
\hline Mesorhoea sexspinosa Stimpson, 1871 & $\begin{array}{l}\text { CN-Fla-GMx-A-GUI-AP-PA-CE-RN-PE-AL-RJ-SP- } \\
\text { PR-RS }\end{array}$ \\
\hline Osachila antillensis Rathbun, 1898 & A-AP-PA-MA-AL \\
\hline $\begin{array}{l}\text { Parthenope (Parthenope) agona } \\
\text { (Stimpson, 1871) }\end{array}$ & $\begin{array}{l}\text { CN-Fla-GMx-A-T-GUI-AP-PA-RN-PB-AL-BA-ES-RJ- } \\
\text { RS }\end{array}$ \\
\hline $\begin{array}{l}\text { P. (Platylambrus) fraterculus } \\
\text { (Stimpson, 1871) }\end{array}$ & CN-Fla-GMx-Yu-A-GUI-AP-PA-MA-RJ-SP-RS \\
\hline P. (P.) pourtalesii (Stimpson, 1871) & MS-Fla-Yu-A-GUI-AP-PA-CE-RJ-PR-RS-URU \\
\hline $\begin{array}{l}\text { P. (P.) serrata (H. Milne Edwards, } \\
\text { 1834) }\end{array}$ & CN-Fla-BM-GMx-A-COL-VEN-GUI-MA-PI-BA-SP \\
\hline $\begin{array}{l}\text { Solenolambrus tenellus Stimpson, } \\
1871\end{array}$ & CN-Fla-GMx-A-PA-RN \\
\hline $\begin{array}{c}\text { Thyrolambrus astroides Rathbun, } 1894 \\
\text { PORTUNIDAE }\end{array}$ & 4CN-A-PA-MA-PI-CE-PB-PE-AL-BA-ES-RJ \\
\hline Callinectes ornatus Ordway, 1863 & $\begin{array}{l}\text { VA-Fla-BM-GMx-A-AC-COL-VEN-T-GUI-AP-MA- } \\
\text { CE-RN-PB-PE-AL-SE-BA-ES-RJ-SP-PR-SC-RS }\end{array}$ \\
\hline
\end{tabular}


Tabela I. (Cont.)

Táxons

Cronius ruber (Lamarck, 1818)

C. tumidulus (Stimpson, 1871)

Portunus anceps (Saussure, 1858)

P. ordwayi (Stimpson, 1860)

P. rufiremus Holthuis, 1959

P. spinicarpus (Stimpson, 1871)

P. spinimanus (Latreille, 1819)

\section{XANTHIDAE}

Actaea acantha (H. Milne Edwards, 1834)

Banareia palmeri (Rathbun, 1894)

Euryozius bouvieri (A. Milne Edwards, PA-MA-PI-CE

1869)

Haxapanopeus paulensis Rathbun, 1930

Lophopanopeus sp.

Melybia thalamita Stimpson, 1871

Micropanope nuttingi (Rathbun, 1898)

M. pusilla A. Milne Edwards, 1881

M. sculptipes Stimpson, 1871

M. urinator (A. Milne Edwards, 1881)

Nanoplax xanthiformis (A. Milne Edwards, 1881)

Panopeus harttii Smith, 1869

Paractaea rufopunctata nodosa

(Stimpson, 1860)

PILUMNIDAE

Pilumnus caribaeus Desbonne \& Schramm, 1867

P. dasypodus Kinsley, 1879

P. diomedeae Rathbun, 1894

P. quoyi H. Milne Edwards, 1834

P. reticulatus Stimpson, 1860 BA BA-ES PE-AL-BA FN SP-PR-SC-RS $\mathrm{SC}$
Atlântico Ocidental

NJ-Fla-GMx-A-AC-COL-VEN-GUI-AP-PA-MA-PICE-BA-RJ-SP-PR-SC-RS

Fla-BM-A-COL-VEN-GUI-PA-MA-PI-CE-RN-PBPE-AL-SE-BA-SP-SC

CN-Fla-BM-A-AC-COL-AP-PA-MA-PI-CE-PE-AL-

MS-Fla-BM-GMx-Yu-A-COL-VEN-GUI-PA-MA-PICE-RN-PB-PE-AL-BA-RJ-FN

GUI-AP-PA

CN-Fla-BM-GMx-A-COL-VEN-T-GUI-AP-PA-CEAL-BA-ES-RJ-SP-SC-RS

NJ-Fla-BM-GMx-A-AC-COL-VEN-T-GUI-AP-MAPE-AL-BA-RJ-SP-PR-SC-RS

Fla-A-COL-GUI-AP-PA-MA-CE-PB-FN-R

Fla-A-VEN-AP-PA-CE-RN-R

CS-Fla-GMx-AC-PA-MA-CE-PB-PE-AL-RJ-SP-PR-SC AP-PA-MA-PI-CE-PB-PE-AL

Fla-A-AC-VEN-PA-MA-PI-CE-RN-PB-PE-AL-SE-

CN-Fla-GMx-Yu-A-COL-AP-PA-MA-PI-CE-RN-PB-

Fla-GMx-A-PA-MA-PI-CE-RN-PB

CN-Fla-GMx-A-PA-MA

CN-Fla-A-PA-MA-PI-CE

CN-Fla-GMx-A-AC-VEN-AP-PA-RJ

Fla-A-COL-MA-PI-CE-RN-PB-PE-AL-BA-RJ-SP-PR-

CN-Fla-GMx-A-COL-VEN-PA-MA-PI-CE-RN-PEAL-SE-BA-ES-RJ-FN-R-URU

Fla-A-VEN-PA-MA-PI-CE-PE-BA-ES-RJ-SP

CN-Fla-GMx-A-AC-COL-VEN-CE-PB-PE-AL-BA-RJ-

Yu-A-GUI-PA-MA-RJ-SP

GUI-AP-PA-MA-PI-CE-RN-PB-PE-AL-BA-ES-RJ-SP-

A-COL-VEN-AP-PA-MA-PE-AL-BA-RJ-SP-PR-SCURU-ARG

Cont. 
Tabela I. (Cont.)

\begin{tabular}{|c|c|}
\hline Táxons & Atlântico Ocidental \\
\hline \multicolumn{2}{|l|}{ GONEPLACIDAE } \\
\hline Acidops cessari (A. Milne Edwards, 1878) & $\mathrm{CE}$ \\
\hline Chasmocarcinus sp. A & CE-AL-SE-BA \\
\hline Chasmocarcinus sp. B & AP-PA-MA-PI-CE-RN-PB \\
\hline Chasmocarcinus sp. C & AP-PA \\
\hline Chasmocarcinus sp. D & AP-PA \\
\hline Cyclopax pinnotheroides Guinot, 1969 & GUI-PA \\
\hline Euryplax nitida Stimpson, 1859 & CN-Fla-BM-GMx-A-PI-CE-RN-PB-PE-AL-BA-SC \\
\hline Pannoplax depressa Stimpson, 1871 & CN-Fla-GMx-Yu-A-AP-PA-MA-PI-CE-PE-RS \\
\hline Specocarcinus sp. & AP-PA-RS \\
\hline \multicolumn{2}{|l|}{ PINNOTHERIDAE } \\
\hline Dissodactylus crinitichelis Moreira, 1901 & $\begin{array}{l}\text { CN-GMx-A-COL-VEN-PA-PB-PE-BA-RJ-SP-PR- } \\
\text { RS-ARG }\end{array}$ \\
\hline Parapinnixa hendersoni Rathbun, 1918 & Fla-GMx-A-COL-VEN-MA-BA-ES-PR \\
\hline Pinnixa cristata Rathbun, 1900 & CN-Fla-GMx-GUI-AP-PA-RN-PE \\
\hline Pinnixa sayana Stimpson, 1860 & AP-PA-PE-RJ-SP-PR-RS \\
\hline Pinnixa sp. A & PA \\
\hline Pinnixa sp. B & MA \\
\hline Pinnixa sp. D & PA-PE \\
\hline Pinnixa sp. G & PA \\
\hline Pinnotheres sp. & MA \\
\hline \multicolumn{2}{|l|}{ GRAPSIDAE } \\
\hline Euchirograpsus antillensis Turkay, 1975 & Fla-A-MA \\
\hline PALICIDAE & \\
\hline $\begin{array}{l}\text { Palicus affinis A. Milne Edwards \& } \\
\text { Bouvier, } 1899\end{array}$ & $\begin{array}{l}\text { Fla-A-GUI-PA-MA-PI-CE-RN-PB-PE-AL-BA-ES- } \\
\text { ARG }\end{array}$ \\
\hline Palicus sp. B & AP-PA \\
\hline Palicus sp. C & $\mathrm{CE}$ \\
\hline P. dentatus (A. Milne Edwards, 1880) & Fla-GMx-A-AP-PA-RS \\
\hline P. sica (A. Milne Edwards, 1880) & CS-Fla-GMx-A-AP-RJ-RS \\
\hline CRYPTOCHIRIDAE & \\
\hline Troglocarcinus corallicola (Verril, 1908) & Fla-BM-A-MA-PE-AL-BA-R \\
\hline
\end{tabular}

Espécies com limites norte e sul fora da área estudada.

Neste grupo foram reunidas 99 espécies. O extremo setentrional e meridional destas foi bastante variável. Desta forma, três espécies apresentaram limite norte em Massachussets; duas em Nova Jersey; uma em Delaware; uma na Virgínia; 42 na Carolina do Norte; três na Carolina do Sul; quatro no norte do Golfo do México; 22 na Flórida; 12 nas Antilhas; três no norte da América do Sul, nas Guianas e no Amapá. Quanto à fronteira meridional, 28 não ultrapassaram o Espírito Santo; 62 apresentaram limite sul entre o Rio de Janeiro e o Rio Grande do Sul; três estenderam-se até o Uruguai e seis até a Argentina. Os braquiúros com este padrão de distribuição e sem registro desde 
as Guianas até algum ponto situado entre $50^{\circ} \mathrm{W}$ e $47^{\circ} \mathrm{W}$, foram representados por 36 espécies. Várias ordens de crustáceos apresentaram também espécies com interrupção no setor guianense, conforme pode ser observado nos trabalhos de COELHO \& RAMOS (1972), COELHO et al. (1977.78, 1980), COELHO \& SANTOS (1980), COELHO \& TORRES (1980, 1987). Espécies com este tipo de distribuição foram denominadas pelos autores precedentes de tropicais disjuntas. Critério análogo caberia para estas 36 espécies de braquiúros estudados. Por outro lado, espécies sem interrupção no setor guianense foram igualmente constatadas pelos autores acima citados, que as denominaram de tropicais contínuas. Na região analisada, os braquiúros com distribuição contínua perfizeram um total de 63 espécies.

\section{CONCLUSÕES}

A fauna braquiurológica da plataforma continental do Norte e Nordeste do Brasil, esteve representada por 113 espécies, 79 gêneros e 15 famílias.

A distribuição das espécies por família foi a seguinte: Majidae (42), Leucosiidae (15), Parthenopidae (13), Xanthidae (13), Goneplacidae (9), Pinnotheridae (9), Portunidae (8), Pilumnidae (5), Palicidae (5), Dorippidae (4), Dromiidae (3), Calappidae (3), Raninidae (2), Grapsidae (1) e Cripthochiridae (1).

A distribuição longitudinal dos braquiúros mostrou que a maioria das espécies (12\%) foi encontrada exclusivamente no Atlântico Ocidental, excetuando-se Cycloes bairdii e Pilumnus reticulatus (Anfi-Americanas); Dromidia antillensis, Euryozius bouvieri e Acidops cessaci (Anfi-Atlânticas) Calappa gallus e Cronius ruber (Circuntropicais) e Thyrolambris astroides (Indo-Pacífica).

Com relação à distribuição latitudinal, foram encontradas 11 espécies com limite sul na área estudada, 17 com fronteira norte na área estudada, seis que não ultrapassaram a área de estudo e $99 \mathrm{com}$ limites norte e sul fora desta área.

\section{REFERÊNCIAS BIBLIOGRÁFICAS}

ABELE, L.G. \& W. KIM. 1986. An ilustratedd guide to the marine Decapod crustaceans of Florida. Tech. Serv. 8 (1): 285-436.

1989. The Decapod crustaceans of the Panama Canal. Smithson. Contrib. Zool., Washington, 482: 22-50.

BORDIN, G. 1987. Brachyura da plataforma continental do Estado do Rio Grande do Sul, Brasil e áreas adjacentes (Crustacea, Decapoda). Iheringia, Ser. Zool., Porto Alegre, 66 (3): 3-32.

BULLIS, H.R. \& J.C. THOMPSON. 1965. Collections by the exploratory fishing vessals Oregon Silver Bay, Combat and Pelican: made during 1956-1960 in the Southwestern North Atlantic. Spec. Sic. Rep. Fish., Washington, 510: $1-130$.

COELHO, P.A. \& A. DO V. BARRETO. 1990. Ocorrência de Acidops cessaci 
(A. Milne Edwards, 1878) (Crustacea, Decapoda) no Nordeste do Brasil. Resumos do Encontro de Zoologia do Nordeste, Aracaju, p.49.

COELHO, P.A. \& M.A. RAMOS. 1972. A constituição e a distribuição da fauna de decápodos do litoral leste da América do Sul entre as latitudes de $5^{\circ} \mathrm{N}$ e $39^{\circ}$ S. Trab. Oceanogr. Univ. Fed. Pernambuco, Recife, 13: 133-236.

- 1980. Crustáceos decápodos da costa do Maranhão, Brasil. Bol. Inst. Oceanogr. São Paulo, São Paulo, 29 (2): 135-138.

- 1986. Sinopse dos crustáceos decápodos brasileiros (famílias Dorippidae e Leucosiidae). Cad. Ômega Univ. Fed. Rur. Pernambuco, Ser. Ci. Aquát., Recife, (2): 67-77.

. 1987/1989. Sinopse dos crustáceos decápodos brasileiros (famílias Dromiidae e Homolidae). Trab. Oceonogr. Univ. Fed. Pernambuco, Recife, 20: $213-218$.

COELHO, P.A.; M. RAMOS-PORTO \& T.C. DOS S. CALADO. 1983. Litoral de Alagoas e Sergipe: Decapoda. An. Soc. Nordest. Zool., Maceió, 1 (1): 133-155.

COELHO, P.A.; M. RAMOS-PORTO \& M.L. KOENING. 1978. Crustáceos marinhos do Brasil, do Uruguai e da Argentina (ao norte do Mar del Plata): considerações biogeográficas. An. Univ. Fer. Rur. Pernambuco, Recife, 2/3: 227-256.

1980. Biogeografia e bionomia dos crustáceos do litoral equatorial brasileiro. Trab. Oceanogr. Univ. Fed. Pernambuco, Recife, 15: 7-138.

COELHO, P.A. \& M.F.A. SANTOS. 1980. Zoogeografia marinha do Brasil. I. Considerações gerais sobre o método e aplicação a um grupo de crustáceos (Paguros: Crustacea, Decapoda, superfamílias Paguroidea e Coenobitoidea). Bolm. Inst. Oceanogr. São Paulo, São Paulo, 29 (2): 139-144.

COELHO, P.A. \& M.F.A. TORRES. 1980. Zoogeografia marinha do Brasil II. Considerações ecológicas e biogeográficas sobre a família Leucosiidae (Decapoda, Brachyura). Rev. Nordest. Biol., João Pessoa, 3: 67-77.

— 1987. Crustacea Stomatopoda: distribuição geográfica no Atlântico Ocidental. An. Soc. Nord. Zool., Teresina, 2 (2): 53-67.

1990. Biogeografia e ecologia das espécies de Inachoidinae na costa atlântica da América do Sul (Crustacea: Decapoda: Majidae). Resumos do XVII Congresso Brasileiro de Zoologia, Londrina, p.9.

FAUSTO-FILHO, J. 1966. Primeira contribuição ao inventário dos crustáceos decapodos marinhos do Nordeste brasileiro. Arq. Est. Biol. Mar. Univ. Fed. Ceará, Fortaleza, 6 (1): 31-37.

1967. Sobre os calapídeos do Norte e Nordeste do Brasil. Arq. Est. Biol. Mar. Univ. Fed. Ceará, Fortaleza, 7 (1): 41-62.

1968. Terceira contribuição ao inventário dos crustáceos decápodos marinhos do Nordeste brasileiro. Arq. Est. Biol. Mar. Univ. Fed. Ceará, Fortaleza, 8 (1): 43-45.

1970. Quarta contribuição ao inventário dos crustáceos decápodos marinhos do Nordeste brasileiro. Arq. Ci. Mar, Fortaleza, 10 (1): 55-60. 
1975. Quarta contribuição ao inventário dos crustáceos decápodos marinhos do Nordeste brasileiro. Arq. Ci. Mar, Fortaleza, 18 (1/2): 63-71. 1978. Crustáceos estomatópodos e decápodos do substrato de lama do Nordeste brasileiro. Arq. Ci. Mar., Fortaleza, 18 (1/2): 63-71. - 1979. Crustáceos estomatópodos e decápodos do substrato de lama do Nordeste brasileiro. Arq. Ci. Mar., Fortaleza, 19 (1/2): 45-56.

FRANKS, J.S.; J.Y. CHRISTMAS; W.L. SILER; R. COMBS; R. WALLER \& C. BURNS. 1972. A study of nectonic and benthic faunas of shallow Gulf of Mexico off the state of Mississipi: as related to some physical, chemical and geological factores. Gulf Res. Rep. 4 (1): 1-148.

FURON, R. 1969. La distribución de los seres. Barcelona, Labor, $3^{\text {a }}$ ed., 162p. GARTH, J.S. 1958. Brachyura of the Pacific coast of America Oxyrhyncha. Atl. Hancock Pac. Exped., Los Angeles, 21 (1): 1-149.

GOEKE, G.D. 1989. Stenorhynchus yangi, a new western atlantic species of arrow crab (Crustacea, Brachyura, Majidae) and a redescription of S. seticornis (Herbst, 1788). Proc. Biol. Soc. Wash. 102 (3): 621-636.

GOMES-CORRÊA, M.M. \& I.N. SILVA-BRUM. 1980. Lista dos crustáceos decápodos e estomatópodos do extremo Norte do Brasil, coletados pelo navio oceanográfico "Almirante Saldanha". Atas Soc. Biol. Rio de Janeiro, 21: $59-64$.

GOUVÊA, E.P. 1980. A carcinofauna do litoral arenoso e arenolodoso de Salvador-BA e áreas adjacentes. Ci. \& Cult., Salvador, 38 (5): 875-883.

1986. A carcinofauna do litoral rochoso de Salvador, BA, e alguns aspectos ecológicos. Ci. \& Cult., Salvador, 38 (2): 346-356.

GUINOT-DUMORTIER, D. 1959. Sur une collection de crustacés (Decapoda Reptantia) de Guyane Française. I. Brachyura (Oxyrhyncha exclus). Bull. Mus. Natl. Hist. Natl., Paris, 31 (5): 423-434.

1960. Sur une collection de crustacés (Decapoda Reptantia) de Guyane Française. II. Brachyura Oxyrhyncha et Macrura. Bull. Mus. Natl. Hist. Natl., Paris, 32 (2): 177-187.

GUNTER, G. \& G.E. HALL. 1965. A biological investigation of the Caloosahatches Estuary of Florida. Gulf Res. Rep., Ocean Springs, Mississipi, 2 (1): 1-71.

HOLTHUIS, L.B. 1959. The Crustacea Decapoda of Suriname (Dutch Guiana). Zool. Verh., Leiden, 44: 161-269.

MANNING, R. \& D.L. FELDER. 1989. The Pinnixa cristata complex in the western Atlantic with description of two new species (Crustacea: Decapoda: Pinnotheridae). Smithson. Contr. Zool., Washington, 473: 1-25.

MANNING, R. \& L.B. HOLTHUIS. 1981. West African Brachyura crabs (Crustacea: Decapoda). Smithson. Contr. Zool., Washington, 306: 1-379.

MARTÍNEZ-IGLESIAS, J.C. \& O. GOMES-HERNÁNDEZ. 1986. Los crustaceos decápodos del Golfo de Batabanó: Brachyura. Inst. Zool. Acad. Ci., La Habana, 332: 2-91.

MELO, G.A.S. DE. 1985. Taxonomia e padrões distribucionais e ecológicos 
dos Brachyura (Crustacea: Decapoda) do litoral sudeste do Brasil. Tese de Doutorado, não publicada, Departamento de Zoologia, Universidade de São Paulo, São Paulo, 215p.

1987. Coleção "Henrique Rodrigues da Costa", doada pela Universidade Federal do Rio de Janeiro para o Museu de Zoologia da Universidade de São Paulo. Não publicado, São Paulo, Museu de Zoologia, Seção de Carcinologia, $19 \mathrm{p}$.

MELO, G.A.S.; V.G. VELOSO \& M.C. DE OLIVEIRA. 1989. A fauna de Brachyura (Crustacea, Decapoda) do litoral do Estado do Paraná: lista preliminar. Nerítica, Pontal do Sul, 4 (1/2): 1-31.

MONOD, T. 1956. Hippidea et Brachyura ouest-africans. Mem. Inst. Fr. Afr. Noire, Paris, (45): 1-674.

OLIVEIRA-FILHO, E.C. DE. 1977. Algas marinhas bentônicas do Brasil.

Tese de Livre Docência, não publicada, Departamento de Botânica, Universidade de São Paulo, São Paulo, 407p.

PEQUEGNAT, W.E. 1970. Deep-water brachyuran crabs, p.1: 171-204. In:

W.E. PEQUEGNAT \& F.A. CHACE JR. (ed.). Contributions on biology of the Gulf of Mexico. A. \& M. University Oceanographic Studies, Houston, 1: 171-204.

PEQUEGNAT, W.E.; L.H. PEQUEGNAT; R.W. FIRTH JR.; B.M. JAMES \& T.W. ROBERTS. 1971. Gulf of mexico deep sea fauna: Decapoda and Euphausiacea. New York, American Geographical Society, Serial atlas of the marine enviroment, 12p.

POWERS, L.W. 1977. A catalogue and bibliography to the crabs (Brachyura) of the Gulf of Mexico. Port Aransas, University of Texas Marine Science Institute, 190p.

RATHBUN, M.J. 1918. The Grapsoid crabs of America. Bull. U.S. Nat. Mus., Washington, 97: 1-461.

1925. The spider crabs of America. Bull. U.S. Nat. Mus, Washington, 129: 1-613.

- 1980. The cancroid crabs of America of the families Euryalidae, Portunidae, Atelecyclidae, Cancridae and Xanthidae. Bull. U.S. Nat. Mus., Washington, 152: 1-609.

- 1937. The Oxystomatous and allied crabs of America. Bull. U.S. Nat. Mus., Washington, 166: 1-278.

RICHMOND, A.E. 1962. The fauna and flora of Horn Island, Mississipi. Gulf Res. Rep., Ocean Springs, Mississipi, 1 (2): 59-106.

RODRÍGUEZ, B.D.G. 1982. Los carangrejos de la familia Portunidae (Decapoda: Brachyura) del Caribe colombiano. An. Inst. Inv. Mar. Punta de Betín, Colombia, 12: 137-184.

RODRÍGUEZ, G/ 1980. Los crustaceos decapodas de Venezuela. Caracas, Instituto Venezolano de Investigaciones Cientificas, 494p.

SOTO, L.A. 1980. Decapod crustacea shelf-fauna of the northeastern Gulf of Mexico. An. Cent. Ci. Mar. Limnol. Univ. Nat. Autón. Mexico 7 (2): 70-110. 
TORRES, F.A. 1988. Biogeografia da família Majidae na costa atlântica da América do Sul (Crustacea, Decapoda). Tese de Mestrado, não publicada, Departamento de Oceanografia, Universidade Federal de Pernambuco, 323p.

VERRIL, A.E. 1908. Decapod crustacea of Bermudas. I. Brachyura and Anomura, their distribution, variation and habits. Trans. Conn. Acad. Arts Sci., New Haven, 13: 299-474.

WILEY, E.O. 1981. Phylogenetics: the theory and practice of phylogenetic systematics. New York, Wiley, 439p.

WILLIAMS, A.B. 1965. Marine decapod crustaceans of the Carolinas. Fish. Bull., Washington, 65 (1): 1-297. 1984. Shrimps, lobsters and crabs of the atlantic coast of the Eastern United States maine to Florida. Washington, Smithsonian Institute, 545p.

Recebido em 23.X.1993; aceito em 26.Il.1994. 